Original paper

\title{
Risk factors predicting nosocomial, healthcare-associated and community-acquired infection in spontaneous bacterial peritonitis and survival outcome
}

\author{
Mayank Jain, Uday Sanglodkar, Jayanthi Venkataraman \\ Global Health City, India
}

\begin{abstract}
Aim of the study: To determine risk factors predicting nosocomial, healthcare-associated and communityacquired infection in spontaneous bacterial peritonitis (SBP) and survival outcome.

Material and methods: This prospective observational study included confirmed cases of cirrhosis with ascites requiring paracentesis, age > 18 years, either gender, any aetiology and Child-Turcotte-Pugh (CTP) stage, with or without cirrhosis-related complications. Patient data included age, gender, co-morbidity, model for end-stage liver disease (MELD) score, CTP score, cirrhosis-related complications, details of previous hospitalization, ascitic tapping and antibiotics instituted. SBP was diagnosed as ascitic fluid polymorphonuclear leucocyte count greater than $250 / \mathrm{mm}^{3}\left(0.25 \times 10^{9} / \mathrm{l}\right)$ and/or culture positivity for a single organism. Statistics - chi square test, Mann-Whitney $U$ test, ANOVA, survival plot. A $p$ value $<0.05$ was statistically significant.

Results: 610 cases fulfilled the criteria for inclusion. 122 (20\%) patients had SBP: community-acquired SBP 37 (30.3\%), nosocomial SBP 19 (16.5\%) and healthcare-associated SBP 66 (54.5\%). The majority were men (106; 86\%) with median age of 51.5 (27-78) years. A significantly higher percentage of community-acquired SBP belonged to CTP class B. Thirtytwo and 7 patients respectively were blood and ascitic fluid culture positive. Significant nosocomial SBP were blood culture positive $(p<0.02)$. The most common isolates were $E$. coli followed by Klebsiella. Survival plot analysis at 3 months showed the worst survival for nosocomial $\operatorname{SBP}(p=0.0009)$.

Conclusions: Prevalence of SBP in our study was 20\%, the majority with healthcare-associated SBP belonging to CTP C. Patients with nosocomial SBP had significant bacteremia with high mortality.
\end{abstract}

Key words: infection, ascites, cirrhosis.

Address for correspondence

Dr. Mayank Jain, Global Health City, 439 Cheran Nagar, 600100, Chennai, India, e-mail: mayank4670@rediffmail.com

\section{Introduction}

The liver is an important site for removal of bloodborne pathogens. In patients with cirrhosis, there is immune system dysfunction characterised by impaired phagocytosis, defective opsonic activity and abnormal neutrophil function. Consequent to this there is a five to sevenfold increase in the risk of bacterial infection in cirrhosis $[1,2]$. Spontaneous bacterial peritonitis
(SBP), an important complication of cirrhosis, is considered as a marker of end stage liver disease and an indication for liver transplant. Previous studies have shown that mortality risk following SBP rises with increasing model for end-stage liver disease (MELD) score [3].

The present study was undertaken to study prevalence, differences and survival outcome following nosocomial (N-SBP), community-acquired (C-SBP) 
and healthcare-associated (H-SBP) SBP infection in decompensated cirrhotic patients.

\section{Material and methods}

This prospective observational study was done at Gleneagles Global Health City, Chennai between September 2016 and February 2018. The study cohort included patients with confirmed liver cirrhosis by either radiological imaging or histology seeking single or multiple paracentesis either during hospitalization or as a day care procedure.

Inclusion criteria: confirmed cirrhosis with ascites requiring diagnostic or therapeutic paracentesis, age $>18$ years, either gender, any aetiology and ChildTurcotte-Pugh (CTP) stage, with or without cirrhosisrelated complications such as sepsis, hepatic encephalopathy, variceal bleed and renal dysfunction.

Exclusion criteria: secondary cause of ascites such as malignant ascites, peritoneal tuberculosis, chylous, biliary, urinary or pancreatic ascites, cirrhotic ascites with partial small bowel obstruction or a perforated viscus.

Patient data (Fig. 1) included age, gender and co-morbidity (diabetes, hypertension, coronary heart disease, dyslipidaemia and hypothyroid state). Details of cirrhosis-related complications such as variceal bleeding, SBP, hepatic encephalopathy, ascites, renal dysfunction and sepsis in the present or in the past, details of previous hospitalization, ascitic tapping (including duration of stay) and antibiotics instituted in the preceding 3 months were noted. MELD and CTP scores were calculated. Baseline laboratory tests included blood counts, serum electrolytes, serum creatinine and liver biochemistry.

Diagnosis of SBP: This was based on either an ascitic fluid polymorphonuclear leucocyte count greater than $250 / \mathrm{mm}^{3}\left(0.25 \times 10^{9} / \mathrm{l}\right)$ and/or culture positivity for a single organism.

Diagnostic paracentesis was performed by the bedside under aseptic conditions. Indications for therapeutic paracentesis were either tense ascites causing abdominal

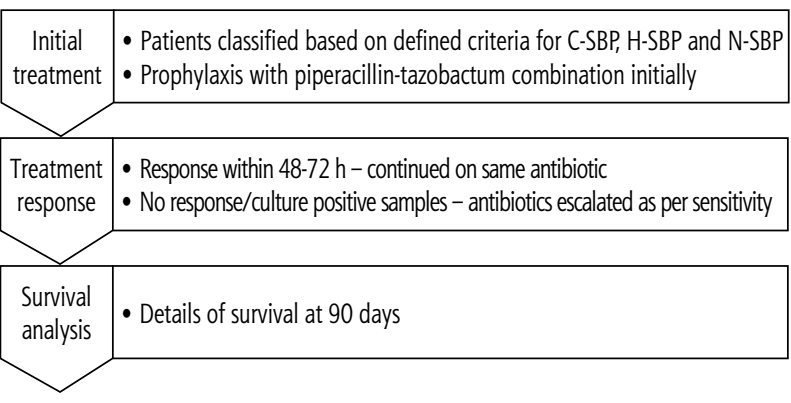

Fig. 1. Study methodology discomfort, breathlessness or a diffuse abdominal pain. Using a $16 \mathrm{G}$ needle, a $20 \mathrm{ml}$ sample was collected in a sterile ethylene diamine tetra acetate (EDTA) preserved tube and analyzed within 3 hours of extraction. The fluid was analyzed for total protein, albumin and total and differential leukocyte count. Giemsa stain was done for identifying bacteria. Another $10 \mathrm{ml}$ of ascitic fluid was inoculated into the blood culture bottle (Bactec 9240) containing trypticase as the broth and observed for the next $72 \mathrm{~h}$. Indications for blood culture were the presence of systemic signs of infection. Site-specific culture of urine, skin and sputum was done for urosepsis, cellulitis and respiratory symptoms respectively. Antibiotic culture pattern was obtained for the major causative organisms: Escherichia coli, Klebsiella and Enterococcus, etc. Antibiotic sensitivity was assessed for cephalosporins, B-lactamase, piperacillin/tazobactam (PIP-TAZ), fluoroquinolones, aminoglycosides, carbapenems, tigecycline, colistin and polymyxin.

Patients with fever, and/or abdominal pain received prophylactic piperacillin/tazobactam in the scheduled dose of $4.5 \mathrm{~g}$ three times a day. The antibiotic was revised subsequently based on the sensitivity report. The antibiotic was also modified when there was no response within $72 \mathrm{~h}$ of introduction of PIP-TAZ or there was an alternative antibiotic sensitivity pattern. The final alternative was introduction of meropenem when there was no response to a specific antibiotic and cultures were also negative.

The primary end point of the study was survival at 3 months.

Community-acquired SBP (C-SBP): SBP occurring within $72 \mathrm{~h}$ of admission to the hospital [4].

Nosocomial SBP (N-SBP): SBP occurring $72 \mathrm{~h}$ after admission to the hospital [5].

Healthcare-associated SBP (HCA-SBP): SBP in admitted patients who were hospitalized in the preceding 90 days of current admission [6].

Multi-drug-resistant bacteria (MDR): These are resistant to 3 or more of the principal antibiotic families, including $\beta$-lactams. The most common bacteria are extended-spectrum $\beta$-lactamase-(ESBL)-producing E. coli, methicillin-resistant Staphylococcus aureus (MRSA), carbapenemase-producing Klebsiella (CPK) and vancomycin-resistant Enterococcus (VRE) [7].

Sample size: 110 subjects [8].

\section{Statistical analysis}

Demographic parameters (age, gender, clinical parameters such as CTP grading, etiology and co-morbidities) were considered as other explanatory variables. Descriptive analysis was analysed by median and range for quantitative variables and frequency and 
proportion for categorical variables. The association between categorical explanatory variables and outcomes was assessed by cross tabulation and comparison of percentages. The chi-square test was used to test statistical significance. Comparison of medians was done using the Mann-Whitney $U$ test and ANOVA. A survival plot was plotted for various variables using the log rank test. A $p$ value $<0.05$ was considered statistically significant.

The study was performed with ethical standards which conform with the Helsinki Declaration of 1975, as revised in 2000 and 2008, confirming human and animal rights. The study was approved by the ethics committee of the institute.

\section{Results}

\section{Baseline characteristics}

Of the 870 patients with cirrhosis and ascites registered during the study period, 610 fulfilled the criteria for inclusion. The majority were men $(106 ; 86 \%)$, with an overall median age of 51.5 (27-78) years. 122 (20\%) patients had SBP: C-SBP in 37 (30.3\%), N-SBP in 19(16.5\%)

Table 1. Comparative analysis of clinical profile in N-SBP, C-SBP and H-SBP

\begin{tabular}{|c|c|c|c|c|}
\hline Parameter & $\mathrm{N}-\mathrm{SBP}(n=19)$ & C-SBP $(n=37)$ & H-SBP $(n=66)$ & $p$ value \\
\hline \multicolumn{5}{|l|}{ Age } \\
\hline Median (range) & $48(29-71)$ & $52(27-71)$ & $51(27-78)$ & 0.32 \\
\hline \multicolumn{5}{|l|}{ Gender } \\
\hline Male & $19(100)$ & $30(82)$ & $57(86)$ & \multirow[t]{2}{*}{0.38} \\
\hline Female & None & $7(18)$ & $9(14)$ & \\
\hline \multicolumn{5}{|l|}{ Co-morbidity } \\
\hline Diabetes & 6 & 14 & 23 & \multirow[t]{3}{*}{0.77} \\
\hline Other comorbidity & 2 & 7 & 8 & \\
\hline No comorbidity & 11 & 16 & 35 & \\
\hline \multicolumn{5}{|l|}{ Aetiology } \\
\hline Alcohol & $8(42.1)$ & $21(56.75)$ & $34(51.5)$ & \multirow[t]{5}{*}{0.43} \\
\hline Cryptogenic & $5(26.3)$ & $11(29.72)$ & $20(30.3)$ & \\
\hline $\mathrm{HBV} / \mathrm{HCV}$ & $4(21)$ & $2(5.4)$ & $5(7.6)$ & \\
\hline Others & $2(10.5)$ & $1(2.7)$ & $6(9.1)$ & \\
\hline Alcohol + Virus & None & $2(5.4)$ & $1(1.5)$ & \\
\hline \multicolumn{5}{|l|}{ CTP Class } \\
\hline$B$ & $2(10.5)$ & $8(21.6)$ & $3(4.5)$ & \multirow[t]{2}{*}{0.03} \\
\hline C & 17 (89.5) & $29(78.4)$ & $63(95.5)$ & \\
\hline \multicolumn{5}{|l|}{ MELD } \\
\hline Median (range) & $21(13-40)$ & $24(13-38)$ & $22.5(12-38)$ & \multirow[t]{3}{*}{0.37} \\
\hline$<15$ & $3(15.8)$ & $3(8.1)$ & 7 (10.6) & \\
\hline$>15$ & $16(84.2)$ & 34 (91.9) & $59(89.4)$ & \\
\hline \multicolumn{5}{|l|}{ Symptomatic SBP } \\
\hline Symptomatic SBP & $3(16)$ & $17(46)$ & $22(33)$ & \multirow[t]{2}{*}{0.07} \\
\hline Asymptomatic SBP & $16(84)$ & $20(54)$ & $44(67)$ & \\
\hline $\mathrm{SBP}+\mathrm{CRC}$ & $15(79)$ & $16(43)$ & $38(58)$ & \multirow[t]{2}{*}{0.04} \\
\hline $\mathrm{SBP}+\mathrm{No} C \mathrm{CRC}$ & $4(21)$ & $21(57)$ & $28(42)$ & \\
\hline \multicolumn{5}{|l|}{ Survival at 3 months } \\
\hline Alive & $4(42.2)$ & $29(78.3)$ & $37(56.1)$ & \multirow[t]{2}{*}{0.00043} \\
\hline Death & $15(78.9)$ & $8(21.6)$ & $29(43.9)$ & \\
\hline
\end{tabular}

CRC - cirrhosis-related complications 
and H-SBP in 66 (54.5\%). Clinically, 81 (65.7\%) patients were asymptomatic, 20 had abdominal pain (16.3\%), abdominal pain with fever and only fever in 11 each (9\%). Previous history of SBP was noted in $9(7 \%)$ cases.

\section{Comparative analysis between N-SBP, C-SBP and $\mathrm{H}-\mathrm{SBP}$}

\section{Clinical profile}

Patients in the three groups were comparable for age, gender, comorbidity and etiological spectrum of cirrhosis. Also there were no significant differences in the clinical presentation. A significantly higher percentage of C-SBP belonged to CTP class $\mathrm{B}$ while the majority of H-SBP and N-SBP were in CTP class $\mathrm{C}$ with higher rates of associated cirrhosis-related complications.
Survival outcome was worst with N-SBP (78.9\% deaths) and least with C-SBP (54\% survival) (Table 1).

\section{Laboratory parameters}

Patients with C-SBP had higher ALT levels $(p<0.05)$. There was no significant difference in the remaining liver biochemistry and renal parameters (Table 2).

\section{Microbiological profile and response to antibiotics}

Thirty-two and 7 patients respectively were blood and ascitic fluid culture positive. There were fewer ascitic fluid culture positive results in N-SBP and H-SBP compared to blood culture. Significant number of cases with N-SBP were blood culture positive $(p<0.02)$

Table 2. Comparison of laboratory parameters in N-SBP, C-SBP, H-SBP

\begin{tabular}{|c|c|c|c|c|}
\hline Parameters & N-SBP $(n=19)$ & C-SBP $(n=37)$ & H-SBP $(n=66)$ & $p$ value \\
\hline \multicolumn{5}{|c|}{ Haemoglobin (gm/dl) } \\
\hline$<12$ & $19(100)$ & $35(94.6)$ & $64(97)$ & \multirow[t]{2}{*}{ NA } \\
\hline$>12$ & 0 & $2(5.4)$ & $2(3.0)$ & \\
\hline \multicolumn{5}{|c|}{ White blood cell count (cells/mm³) } \\
\hline$<4000$ & $3(15.8)$ & $1(2.7)$ & $5(7.6)$ & \multirow[t]{3}{*}{0.27} \\
\hline $4000-10000$ & $9(47.4)$ & $14(37.8)$ & $22(33.3)$ & \\
\hline$>10000$ & $7(36.8)$ & $22(54.5)$ & $39(59.0)$ & \\
\hline \multicolumn{5}{|c|}{ Platelet count (cells/mm³) } \\
\hline$<150000$ & $14(73.7)$ & $26(70.3)$ & $49(74.3)$ & \multirow[t]{2}{*}{0.9} \\
\hline$>150000$ & $5(26.3)$ & $11(29.7)$ & $17(25.7)$ & \\
\hline \multicolumn{5}{|c|}{ INR (median, range) } \\
\hline 1.01 & $2.43(1.35-5.7)$ & $1.8(1.3-2.9)$ & $1.9(1.19-3.92)$ & \\
\hline \multicolumn{5}{|l|}{ S. bilirubin (mg/dl) } \\
\hline$<1.2$ & $1(5.2)$ & $2(5.4)$ & $2(3)$ & \multirow[t]{2}{*}{0.81} \\
\hline$>1.2$ & $18(94.8)$ & $35(94.6)$ & $64(97)$ & \\
\hline \multicolumn{5}{|l|}{ AST (U/I) } \\
\hline$<30$ & $3(15.8)$ & $8(21.6)$ & $10(15.1)$ & \multirow[t]{2}{*}{0.69} \\
\hline$>30$ & $16(84.2)$ & $29(78.4)$ & $56(84.9)$ & \\
\hline \multicolumn{5}{|l|}{$\mathrm{ALT}(\mathrm{U} / \mathrm{I})$} \\
\hline$<30$ & $3(15.8)$ & $1(2.7)$ & $13(19.7)$ & \multirow[t]{2}{*}{0.05} \\
\hline$>30$ & $16(84.2)$ & $36(97.3)$ & $53(80.3)$ & \\
\hline \multicolumn{5}{|l|}{ Albumin (gm/dl) } \\
\hline$<3.5$ & $17(89.5)$ & $36(97.3)$ & $65(98.5)$ & \multirow[t]{2}{*}{0.14} \\
\hline$>3.5$ & $2(10.5)$ & $1(2.7)$ & $1(1.5)$ & \\
\hline \multicolumn{5}{|c|}{ S. creatinine (mg/dl) } \\
\hline$<1.2$ & $3(15.8)$ & $16(43.2)$ & $19(28.8)$ & \multirow[t]{2}{*}{0.09} \\
\hline$>1.2$ & $16(84.2)$ & $21(56.8)$ & 47 (71.2) & \\
\hline
\end{tabular}


Table 3. Culture positivity in ascitic fluid and blood in N-SBP, C-SBP, H-SBP

\begin{tabular}{|c|c|c|c|c|c|}
\hline & Outcome & $\begin{array}{c}\text { N-SBP (No. \%) } \\
19(16 \%)\end{array}$ & $\begin{array}{c}\text { C-SBP (No. \%) } \\
37(30 \%)\end{array}$ & $\begin{array}{c}\text { H-SBP (No. \%) } \\
66(54 \%)\end{array}$ & $p$ value \\
\hline \multirow[t]{2}{*}{ Ascitic fluid } & Positive & $3(15.8)$ & None & $4(6.1)$ & 0.71 \\
\hline & Negative & 16 (84.2) & None & $66(93.9)$ & \\
\hline \multirow[t]{2}{*}{ Blood } & Positive & $8(42.1)$ & $4(10.8)$ & $20(30.3)$ & 0.02 \\
\hline & Negative & $11(57.9)$ & 33 (89.2) & 46 (69.6) & \\
\hline
\end{tabular}

Table 4. Bacterial isolates and MDR status in blood and ascitic fluid in N-SBP, C-SBP, H-SBP

\begin{tabular}{|c|c|c|c|c|c|c|c|}
\hline & E. coli & Klebsiella & Acinetobacter & Salmonella & Staphylococcus & Enterococcus & Enterobacter \\
\hline \multicolumn{8}{|c|}{ Ascitic fluid culture (7) } \\
\hline N-SBP & - & - & 1 (MDR) & $1(S)$ & $1(S)$ & - & - \\
\hline C-SBP & - & - & - & - & - & - & - \\
\hline H-SBP & $3(S)$ & $1(S)$ & - & - & - & - & - \\
\hline \multicolumn{8}{|c|}{ Blood culture (32) } \\
\hline N-SBP & $3(\mathrm{~S}) ; 2$ (MDR) & 1 (S); 1 (MDR) & - & - & - & - & 1 (MDR) \\
\hline C-SBP & $1(S)$ & 1 (MDR) & - & - & - & $1(S)$ & $1(S)$ \\
\hline H-SBP & $6(S) ; 9$ (MDR) & 1 (S); 3 (MDR) & - & - & - & 1 (MDR) & - \\
\hline
\end{tabular}

S- pan-sensitive, MDR - multi-drug-resistant

(Table 3). The most common isolates were E. coli followed by Klebsiella in both ascitic fluid and blood culture with significant MDR for E. coli and in H-SBP (Table 4).

Antibiotic response: N-SBP most often required PIP-TAZ in combination with culture-sensitive antibiotic, while significant $\mathrm{C}-\mathrm{SBP}$ and $\mathrm{H}-\mathrm{SBP}$ responded to empirical PIP-TAZ $(p<0.0001)$. Fewer C-SBP required meropenem (Table 5).

Survival plot analysis at 3 months (Fig. 2) showed the worst survival for N-SBP $(p=0.0009)$ with better survival for $\mathrm{H}-\mathrm{SBP}$ and $\mathrm{C}-\mathrm{SBP}$.

\section{Discussion}

The prevalence of SBP in our study was $20 \%$, similar to other reports in the literature, where the prevalence has ranged from $3 \%$ to $25 \%[9,10]$. Two studies from India have reported a prevalence of $22 \%$ [11] and $30 \%$ [12]. There are very few studies from India that have compared N-SBP, H-SBP and C-SBP in cirrhotic patients. In our study of 122 patients, 19 (16.5\%) had N-SBP, 37 (30.3\%) C-SBP, and 66 (54.5\%) had H-SBP. In a recent study by Balaraju et al. [13], the prevalence of C-SBP was $87 \%$ and $13 \%$ for N-SBP; C-SBP was comparatively low in our study (Table 6). Ours is a quaternary referral centre for liver transplant and the majority of the patients coming to our centre had been treated elsewhere, resulting in a higher prevalence of H-SBP.
Table 5. Antibiotic response in N-SBP, C-SBP and H-SBP

\begin{tabular}{|c|c|c|c|c|}
\hline $\begin{array}{l}\text { Response } \\
\text { to antibiotic }\end{array}$ & $\begin{array}{c}\text { N-SBP } \\
19(16 \%)\end{array}$ & $\begin{array}{c}\text { C-SBP } \\
37(30 \%)\end{array}$ & $\begin{array}{c}\text { H-SBP } \\
66(54 \%)\end{array}$ & $p$ value \\
\hline Empirical PIP-TAZ & $3(15.8)$ & $27(73)$ & $33(50)$ & 0.0001 \\
\hline PIP-TAZ + others & $12(63.2)$ & $6(16.2)$ & $14(21.2)$ & \\
\hline Meropenem & $4(21)$ & $4(10.8)$ & $19(28.8)$ & \\
\hline
\end{tabular}

Nearly three fifth of patients were asymptomatic. This observation highlights the fact that SBP may not have a clinically overt presentation in a significant proportion of cirrhotics and a high index of suspicion needs to be maintained with an absolute need for an ascitic fluid examination at each visit.

Clinically, there was no distinction between the 3 types of SBP acquisition. Almost a third of each group had either fever or abdominal pain as presenting complaints. This observation is similar to those made by Jain et al. [14]. In a study by Cheong et al., fever was common in N-SBP and abdominal pain in C-SBP [15].

Of 122 patients, 32 (26\%) patients were blood culture positive, most frequent in N-SBP (42\%) followed by H-SBP (30\%) and C-SBP (10\%). Only 7(6\%) were ascitic culture positive. Baijal et al. [16] and Mohammad et al. [17] reported a much higher positivity at $15 \%$ and $25 \%$ respectively. The low culture yield in the present study may be related to prior antibiotic exposure in our cases [18]. 
Table 6. Comparison of previous Indian studies on SBP with present studies

\begin{tabular}{lccc}
\hline & Baijal et al. [16] & Balaraju et al. [13] & Present study \\
\hline No. of patients & $n=420$ & $n=162$ & $n=122$ \\
\hline Type of study (all prospective) & Western and Central India: 6 centres & Single centre & Single centre \\
\hline C-SBP & $8(24 \%)$ & $141(87 \%)$ & $37(30.3 \%)$ \\
N-SBP & $9(27.7 \%)$ & $21(13 \%)$ & $19(16.5 \%)$ \\
H-SBP & $16(48.2 \%)$ & None & $66(54.2 \%)$ \\
\hline Ascitic fluid culture & $15 \%$ & $22 \%$ & $6 \%$ \\
\hline Gram-negative & $54 \%$ & $100 \%$ & $86 \%$ \\
Isolates & E. coli, Klebsiella, Pseudomonas & E. coli, Klebsiella, & E. coli, Klebsiella, Acinetobacter \\
& & non-fermenting GNB & \\
\hline Gram-positive & $46 \%$ & No information & NA \\
Isolates & Not known & NA & S. aureus: $14 \%$ \\
MDR & $1.7 \%$ & 6 months & $44 \%$ \\
\hline Survival & 30 days & 6 mo: $59 \%$ & 3 months \\
\hline Mortality & 1 mo: $23.5 \%$ & & 3 mo: $43 \%$ \\
\hline
\end{tabular}

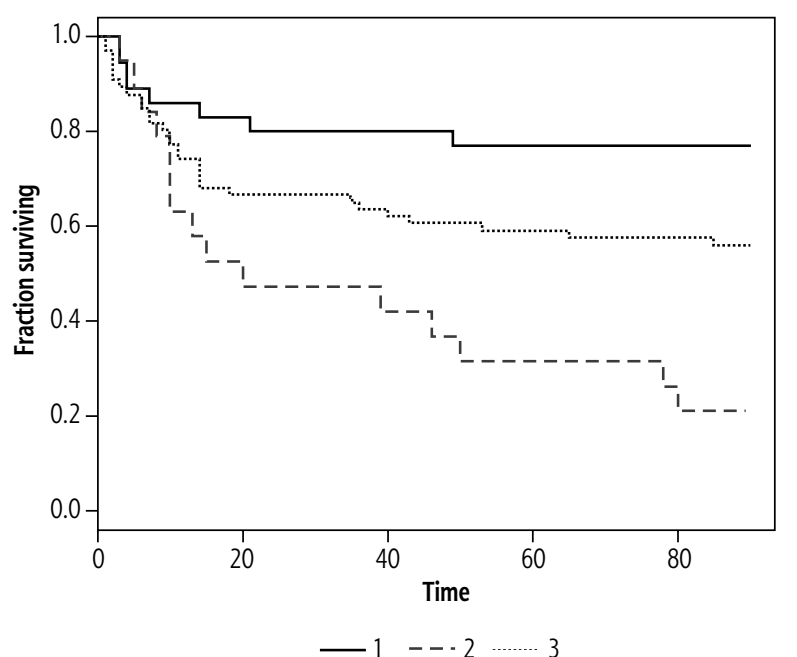

Fig. 2. Survival plot for C-SBP (1), N-SBP (2) and H-SBP (3) using log rank test

Ascitic fluid culture positivity was very low in our series. Though the organisms isolated from ascitic fluid were similar to those reported in other studies from India $[13,16,19]$, the overall culture positivity was low, possibly due to prior antibiotic exposure.

Thirty-two patients had positive blood cultures. An earlier report observed that $33 \%$ to $57 \%$ of patients with neutrocytic ascites may have positive blood culture, indicating bacteraemia [20] with worse outcomes. Acharya et al. [21] also made similar observations. Seventeen (44\%) isolates in our series were multidrug resistant organisms, similar to reports by Baijal et al. [16] and relatively low compared to an earlier study from our centre [18].

The mortality at 3 months in our study was intermediate, as reported by 2 other studies (Table 6).
Summing up, the prevalence of SBP in our study was $20 \%$, the majority with healthcare-associated SBP belonging to CTP C. Patients with nosocomial SBP had significant bacteremia with high mortality.

\section{Limitations of the study}

Though the sample size was adequate in our series, $50 \%$ of our patients had H-SBP with fewer cases in the other 2 groups. Ascitic fluid culture yield was very low, largely related to more patients with healthcare-associated SBP who would have received antibiotics in the preceding 3 months.

\section{Disclosure}

Authors report no conflict of interest.

\section{References}

1. Taneja SK, Dhiman RK. Prevention and management of bacterial infections in cirrhosis. Int J Hepatol 2011; 2011: 784540.

2. Baijal R, Praveenkumar HR, Amarapurkar DN, et al. Prevalence of tuberculosis in patients with cirrhosis of liver in western India. Trop Doctor 2010; 40: 163-164.

3. Jain M, Baijal R, Jaiswal SP. Correlation between MELD score and SBP in patients with cirrhosis and ascites. Int J Curr Adv Res 2017; 6: 5682-5685.

4. Chon YE, Kim SU, Lee CK, et al. Community-acquired vs. nosocomial spontaneous bacterial peritonitis in patients with liver cirrhosis. Hepatogastroenterology 2014; 61: 2283-2290.

5. Venditti M, Falcone M, Corrao S, et al. Study Group of the Italian Society of Internal Medicine (outcomes of patients hospitalized with community-acquired, healthcare associated and hospital-acquired pneumonia). Ann Intern Med 2009; 150: 19-26. 
6. Merli M, Lucidi C, Giannelli V, et al. Cirrhotic patients are at risk for health care-associated bacterial infections. Clin Gastroenterol Hepatol 2010; 8: 979-985.

7. Magiorakos AP, Srinivasan A, Carey RB, et al. Multidrug-resistant, extensively drug-resistant and pan drug-resistant bacteria: an international expert proposal for interim standard definitions for acquired resistance. Clin Microbiol Infect 2012; 18 : 268-281.

8. Daniel WW. Biostatistics: a foundation for analysis in the health sciences. $7^{\text {th }}$ ed. John Wiley \& Sons, New York 1999.

9. Evans TL, Kim WR, Joun JP, Kamath PS. Spontaneous bacterial peritonitis in asymptomatic out patients with cirrhotic ascites. Hepatology 2003; 37: 897-901.

10. Syed VA, Ansari JA, Karki P, et al. Spontaneous bacterial peritonitis (SBP) in cirrhotic ascites: A prospective study in a tertiary care hospital, Nepal. Kathmandu University Med J 2007; 5: 48-59.

11. Amarapurkar DN, Viswanathan N, Parikh SS, et al. Prevalence of spontaneous bacterial peritonitis. J Assoc Phys India 1992; 40: 236-238

12. Puri AS, Puri J, Ghoshal UC, et al. Frequency, microbial spectrum and outcome of spontaneous bacterial peritonitis. Indian J Gastroenterol 1996; 15: 86-89.

13. Balaraju G, Patil M, Krishnamurthy AC, et al. Comparative study of community acquired and nosocomial spontaneous bacterial peritonitis and its variants in 150 patients. J Clin Exp Hepatol 2017; 7: 215-221.

14. Jain AK, Sircar S, Jain M, et al. Acute febrile illness in cirrhosis thinking beyond spontaneous bacterial peritonitis. Trop Doctor 2012; 42: 200-202.

15. Cheong HS, Kang CI, Lee JA, et al. Clinical significance and outcome of nosocomial acquisition of spontaneous bacterial peritonitis in patients with liver cirrhosis. Clin Infect Dis 2009; 48: 1230-1236.

16. Baijal R, Amarapurkar D, Praveen Kumar HR, et al. A multicenter prospective study of infections related morbidity and mortality in cirrhosis of liver. Indian J Gastroenterol 2014; 33: 336-342.

17. Mohammad AN, Yousef LM, Mohamed HS. Prevalence and predictors of spontaneous bacterial peritonitis: does low zinc level play any role? Al-AzharAssiut Med J 2016; 14: 37-42.

18. Jain M, Vaghese J, Balaji G, et al. An insight into antibiotic resistance to bacterial infection in chronic liver disease. J Clin Exp Hepatol 2017; 7: 305-309.

19. Purohit PH, Malek SS, Desai KJ, Sadadia M. A study of bacteriological profile of ascitic fluid in suspected clinical cases of spontaneous bacterial peritonitis at a tertiary care hospital in India. Inter J Med Sci Public Health 2015; 4: 4.

20. Thomas V. Routine analysis of cirrhotic ascites for evidence of infection - not worth the effort. Indian J Gastroenterol 2011; 30: 201-203.

21. Shalimar, Acharya SK. Difficult to treat spontaneous bacterial peritonitis. Trop Gastroenterol 2013; 34: 7-13. 